\title{
ON THE SET OF DISTANCES BETWEEN TWO SETS OVER FINITE FIELDS
}

\author{
IGOR E. SHPARLINSKI
}

Received 13 March 2006; Revised 12 May 2006; Accepted 9 July 2006

We use bounds of exponential sums to derive new lower bounds on the number of distinct distances between all pairs of points $(\mathbf{x}, \mathbf{y}) \in \mathscr{A} \times \mathscr{B}$ for two given sets $\mathscr{A}, \mathscr{B} \in \mathbb{F}_{q}^{n}$, where $\mathbb{F}_{q}$ is a finite field of $q$ elements and $n \geq 1$ is an integer.

Copyright (c) 2006 Hindawi Publishing Corporation. All rights reserved.

\section{Introduction}

For a ring $\mathscr{R}$ and two finite sets $\mathscr{A}, \mathscr{B} \subseteq \mathscr{R}^{n}$, we denote by $\Gamma\left(\mathscr{R}^{n}, \mathscr{A}, \mathscr{B}\right)$ the number of distinct distances between all pairs of points $(\mathbf{x}, \mathbf{y}) \in \mathscr{A} \times \mathscr{B}$, that is,

$$
\Gamma\left(\mathscr{R}^{n}, \mathscr{A}, \mathscr{B}\right)=|\{d(\mathbf{x}, \mathbf{y}) \mid(\mathbf{x}, \mathbf{y}) \in \mathscr{A} \times \mathscr{B}\}|
$$

where for $\mathbf{x}=\left(x_{1}, \ldots, x_{n}\right), \mathbf{y}=\left(y_{1}, \ldots, y_{n}\right) \in \mathscr{R}^{n}$ we define

$$
d(\mathbf{x}, \mathbf{y})=\sum_{j=1}^{n}\left(x_{j}-y_{j}\right)^{2}
$$

In the case $\mathscr{A}=\mathscr{B}$ the problem of estimating $\Gamma\left(\mathscr{R}^{n}, \mathscr{A}, \mathscr{A}\right)$ is well known. In particular, the Erdös distance conjecture asserts that over the real numbers, that is, for $\mathscr{R}=\mathbb{R}$, the bound

$$
\Gamma\left(\mathbb{R}^{n}, \mathscr{A}, \mathscr{A}\right) \geq c(\varepsilon)|\mathscr{A}|^{2 / n-\varepsilon}
$$

holds for an arbitrary $\varepsilon>0$ and any finite set $\mathscr{A} \in \mathbb{R}^{n}$, where $c(\varepsilon)>0$ depends only on $\varepsilon$. Despite that there are some very interesting lower bounds on $\Gamma\left(\mathbb{R}^{n}, \mathscr{A}, \mathscr{A}\right)$, this conjecture is still widely open in any dimension including $n=2$. For some recent achievements and generalisations, see [1-6] and references therein.

Iosevich and Rudnev [4] have recently considered this problem for sets over finite fields (again for $\mathscr{A}=\mathscr{B}$ ) and obtained several very interesting results. 
2 On the set of distances between two sets over finite fields

The case of arbitrary sets $\mathscr{A}, \mathscr{B} \in \mathbb{F}_{q}^{n}$ has recently been studied in [8], where the lower bound

$$
\Gamma\left(\mathbb{F}_{q}^{n}, \mathscr{A}, \mathscr{B}\right)>q-\frac{q^{n+2}}{|\mathscr{A}||\mathscr{B}|}
$$

is given (which in some special case is new even for $\mathscr{A}=\mathscr{B}$ ). In particular, it is nontrivial for $|\mathscr{A}||\mathscr{B}|>q^{n+1}$. The method of [8] rests on a new bound of exponential sums over the set of distances. Here we use this bound in a slightly different way to derive an improvement of (1.4), which is nontrivial for $|\mathscr{A}||\mathscr{B}|>q^{n}$.

In fact, one can easily adjust the method of [4] to the case of distinct sets $\mathscr{A}$ and $\mathscr{B}$, or in fact derive a lower bound on $\Gamma\left(\mathbb{F}_{q}^{n}, \mathscr{A}, \mathscr{B}\right)$ from already existing results of [4]. Such bounds are usually stronger than the bound of this work. However in some extremal cases our approach leads to a bound of the same order of magnitude which has completely explicit (and perhaps better than those one can extract from [4]) constants. For example, one can derive from [4] that if $|\mathscr{A}||\mathscr{B}|>C q^{n+1}$, then $\Gamma\left(\mathbb{F}_{q}^{n}, \mathscr{A}, \mathscr{B}\right)=q$, provided that $C$ is sufficiently large.

Furthermore, as in [8], given $n$ polynomials $f_{j}(X, Y) \in \mathbb{F}_{q}[X, Y], j=1, \ldots, n$, we define the generalised distance

$$
d_{\mathbf{f}}(\mathbf{x}, \mathbf{y})=\sum_{j=1}^{n} f_{j}\left(x_{j}, y_{j}\right)
$$

where $\mathbf{f}=\left(f_{1}, \ldots, f_{n}\right)$.

Now, for two sets $\mathscr{A}, \mathscr{B} \subseteq \mathbb{F}_{q}^{n}$, we define

$$
\Gamma_{\mathbf{f}}\left(\mathbb{F}_{q}^{n}, \mathscr{A}, \mathscr{B}\right)=\left|\left\{d_{\mathbf{f}}(\mathbf{x}, \mathbf{y}) \mid \mathbf{x} \in \mathscr{A}, \mathbf{y} \in \mathscr{B}\right\}\right|
$$

In the special case of the Euclidean distance function $\mathbf{f}_{0}=\left(f_{1,0}, \ldots, f_{n, 0}\right)$, where $f_{j, 0}(X, Y)=$ $(X-Y)^{2}, j=1, \ldots, n$, we simply have

$$
\Gamma_{\mathbf{f}_{0}}\left(\mathbb{F}_{q}^{n}, \mathscr{A}, \mathscr{B}\right)=\Gamma\left(\mathbb{F}_{q}^{n}, \mathscr{A}, \mathscr{B}\right) .
$$

In particular, under some conditions on $\mathbf{f}$, the bound

$$
\Gamma_{\mathbf{f}}\left(\mathbb{F}_{q}^{n}, \mathscr{A}, \mathscr{B}\right)=q+O\left(\frac{q^{3 n / 2+2}}{|\mathscr{A}||\mathscr{B}|}\right)
$$

has been given in [8]. Here we show that the power of $q$ in the error term can be lowered to $q^{3 n / 2+1}$.

\section{Euclidean distances}

We start with the case of Euclidean distances and improve the bound (1.4).

Theorem 2.1. For arbitrary sets $\mathscr{A}, \mathscr{B} \subseteq \mathbb{F}_{q}^{n}$,

$$
\Gamma\left(\mathbb{F}_{q}^{n}, \mathscr{A}, \mathscr{B}\right)>\frac{|\mathscr{A}||\mathscr{B}| q}{q^{n+1}+|\mathscr{A}||\mathscr{B}|} .
$$


Proof. Let $\chi$ be a nontrivial additive character of $\mathbb{F}_{q}$ (see [7] for basis properties of additive characters). In particular, we recall the identity

$$
\sum_{s \in \mathbb{F}_{q}} \chi(s t)= \begin{cases}0 & \text { if } t \in \mathbb{F}_{q}^{*} \\ q & \text { if } t=0\end{cases}
$$

As in [8], we consider character sums

$$
S(a, \mathscr{A}, \mathscr{B})=\sum_{\mathbf{x} \in \mathscr{A}} \sum_{\mathbf{y} \in \mathscr{B}} \chi(a d(\mathbf{x}, \mathbf{y})), \quad a \in \mathbb{F}_{q}
$$

where as before $d(\mathbf{x}, \mathbf{y})$ is given by (1.2).

Our principal tool is the upper bound

$$
|S(a, \mathscr{A}, \mathscr{B})| \leq \sqrt{|\mathscr{A}||\mathscr{B}| q^{n}},
$$

which is established in [8] for any $a \in \mathbb{F}_{q}^{*}$.

For $\lambda \in \mathbb{F}_{q}$, we denote by $N(\lambda)$ the number of representations $\lambda=d(\mathbf{x}, \mathbf{y})$ with $(\mathbf{x}, \mathbf{y}) \in$ $\mathscr{A} \times \mathscr{B}$.

Then by (2.2) we have

$$
N(\lambda)=\frac{1}{q} \sum_{\mathbf{x} \in \mathscr{A}} \sum_{\mathbf{y} \in \mathscr{B}} \frac{1}{q} \sum_{a \in \mathbb{F}_{q}} \chi(a(d(\mathbf{x}, \mathbf{y})-\lambda))=\frac{1}{q} \sum_{a \in \mathbb{F}_{q}} \chi(-a \lambda) S(a, \mathscr{A}, \mathscr{B}) .
$$

Hence,

$$
\begin{aligned}
\sum_{\lambda \in \mathbb{F}_{q}} N(\lambda)^{2} & =\frac{1}{q^{2}} \sum_{\lambda \in \mathbb{F}_{q}} \sum_{a, b \in \mathbb{F}_{q}} \chi((b-a) \lambda) S(a, \mathscr{A}, \mathscr{B}) \overline{S(b, \mathscr{A}, \mathscr{B})} \\
& =\frac{1}{q^{2}} \sum_{a, b \in \mathbb{F}_{q}} S(a, \mathscr{A}, \mathscr{B}) \overline{S(b, \mathscr{A}, \mathscr{B})} \sum_{\lambda \in \mathbb{F}_{q}} \chi((b-a) \lambda) \\
& =\frac{1}{q} \sum_{a \in \mathbb{F}_{q}}|S(a, \mathscr{A}, \mathscr{B})|^{2},
\end{aligned}
$$

since by (2.2) the sum over $\lambda$ vanishes unless $a=b$.

We now use the bound (2.4) for $a \in \mathbb{F}_{q}^{*}$ and the trivial bound $|S(a, \mathscr{A}, \mathscr{B})| \leq|\mathscr{A}||\mathscr{B}|$ for $a=0$, getting

$$
\sum_{\lambda \in \mathbb{F}_{q}} N(\lambda)^{2}<|\mathscr{A}||\mathscr{B}| q^{n}+|\mathscr{A}|^{2}|\mathscr{B}|^{2} q^{-1}
$$

Clearly

$$
\sum_{\lambda \in \mathbb{F}_{q}} N(\lambda)=|\mathscr{A}||\mathscr{B}| .
$$


4 On the set of distances between two sets over finite fields

Now by the Cauchy inequality we derive

$$
\begin{aligned}
(|\mathscr{A}||\mathscr{B}|)^{2} & =\left(\sum_{\lambda \in \mathbb{F}_{q}} N(\lambda)\right)^{2} \leq \Gamma\left(\mathbb{F}_{q}^{n}, \mathscr{A}, \mathscr{B}\right) \sum_{\lambda \in \mathbb{F}_{q}} N(\lambda)^{2} \\
& <\Gamma\left(\mathbb{F}_{q}^{n}, \mathscr{A}, \mathscr{B}\right)\left(|\mathscr{A}||\mathscr{B}| q^{n}+|\mathscr{A}|^{2}|\mathscr{B}|^{2}, q^{-1}\right),
\end{aligned}
$$

which implies the desired result.

\section{Generalised distances}

We now use similar arguments to improve the bound (1.8).

Theorem 3.1. Let $\mathbf{f}=\left(f_{1}, \ldots, f_{n}\right)$, where each of the polynomials $f_{j}(X, Y) \in \mathbb{F}_{q}[X, Y], j=$ $1, \ldots, n$, is of degree at most $k$ and is not of the form $f_{j}(X, Y)=g_{j}(X)+h_{j}(Y)$ with $g_{j}(X) \in$ $\mathbb{F}_{q}[X], h_{j}(Y) \in \mathbb{F}_{q}[Y]$. Then, for arbitrary sets $\mathscr{A}, \mathscr{B} \subseteq \mathbb{F}_{q}^{n}$,

$$
\Gamma_{\mathbf{f}}\left(\mathbb{F}_{q}^{n}, \mathscr{A}, \mathscr{B}\right)=q+O\left(\frac{q^{3 n / 2+1}}{|\mathscr{A}|\left|\mathscr{S}_{3}\right|}\right)
$$

Proof. Here, instead of the bound (2.4), we use the bound

$$
\left|S_{\mathbf{f}}(a, \mathscr{A}, \mathscr{B})\right|=O\left(\sqrt{|\mathscr{A}||\mathscr{B}| q^{3 n / 2}}\right), \quad a \in \mathbb{F}_{q}^{*}
$$

which is established in [8] for the character sums

$$
S_{\mathbf{f}}(a, \mathscr{A}, \mathscr{B})=\sum_{\mathbf{x} \in \mathscr{A}} \sum_{\mathbf{y} \in \mathscr{B}} \chi\left(a d_{\mathbf{f}}(\mathbf{x}, \mathbf{y})\right), \quad a \in \mathbb{F}_{q}
$$

where $d_{\mathbf{f}}(\mathbf{x}, \mathbf{y})$ is given by $(1.5)$.

Let $N_{\mathbf{f}}(\lambda)$ be the number of solutions to the equation

$$
d_{\mathbf{f}}(\mathbf{x}, \mathbf{y})=\lambda, \quad \mathbf{x} \in \mathscr{A}, \mathbf{y} \in \mathscr{B}
$$

As in the proof of Theorem 2.1, using (3.2) instead of (2.4), we deduce

$$
\sum_{\lambda \in \mathbb{F}_{q}} N_{\mathbf{f}}(\lambda)^{2}=\frac{1}{q} \sum_{a \in \mathbb{F}_{q}}|S(a, \mathscr{A}, \mathscr{B})|^{2}=|\mathscr{A}|^{2}|\mathscr{B}|^{2} q^{-1}+O\left(|\mathscr{A}||\mathscr{B}| q^{3 n / 2}\right)
$$

As before, we also have

$$
\sum_{\lambda \in \mathbb{F}_{q}} N_{\mathbf{f}}(\lambda)=|\mathscr{A}||\mathscr{B}|
$$


and by the Cauchy inequality we derive

$$
\begin{aligned}
(|\mathscr{A}||\mathscr{B}|)^{2} & =\left(\sum_{\lambda \in \mathbb{F}_{q}} N(\lambda)\right)^{2} \leq \Gamma\left(\mathbb{F}_{q}^{n}, \mathscr{A}, \mathscr{B}\right) \sum_{\lambda \in \mathbb{F}_{q}} N(\lambda)^{2} \\
& <\Gamma\left(\mathbb{F}_{q}^{n}, \mathscr{A}, \mathscr{B}\right)\left(|\mathscr{A}|^{2}|\mathscr{S}|^{2} q^{-1}+O\left(|\mathscr{A}||\mathscr{B}| q^{3 n / 2}\right)\right),
\end{aligned}
$$

which implies the desired result.

\section{Acknowledgments}

The author is very grateful to Alex Iosevich for many useful discussions and encouragement, in particular, for clarifying how the results and methods of [4] can be modified to incorporate the case of distinct sets $\mathscr{A} \neq \mathscr{B}$. During the preparation of this note, the author was supported in part by ARC Grant DP0556431.

\section{References}

[1] M. B. Erdogan, A bilinear Fourier extension theorem and applications to the distance set problem, International Mathematics Research Notices 2005 (2005), no. 23, 1411-1425.

[2] S. Hofmann and A. Iosevich, Circular averages and Falconer/Erdös distance conjecture in the plane for random metrics, Proceedings of the American Mathematical Society 133 (2005), no. 1, 133143.

[3] A. Iosevich and I. Łaba, Distance sets of well-distributed planar point sets, Discrete \& Computational Geometry 31 (2004), no. 2, 243-250.

[4] A. Iosevich and M. Rudnev, Erdös distance problem in vector spaces over finite fields, to appear in Transactions of the American Mathematical Society.

[5] __ Spherical averages, distance sets, and lattice points on convex surfaces, preprint, 2005.

[6] N. H. Katz and G. Tardos, A new entropy inequality for the Erdös distance problem, Towards a Theory of Geometric Graphs, Contemp. Math., vol. 342, American Mathematical Society, Rhode Island, 2004, pp. 119-126.

[7] R. Lidl and H. Niederreiter, Finite Fields, Encyclopedia of Mathematics and Its Applications, vol. 20, Cambridge University Press, Cambridge, 1997.

[8] I. E. Shparlinski, On some generalisations of the Erdös distance problem over finite fields, Bulletin of the Australian Mathematical Society 73 (2006), no. 2, 285-292.

Igor E. Shparlinski: Department of Computing, Macquarie University, Sydney, NSW 2109, Australia E-mail address: igor@ics.mq.edu.au 


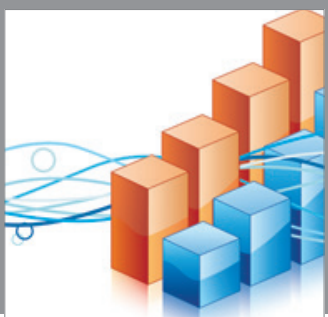

Advances in

Operations Research

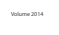

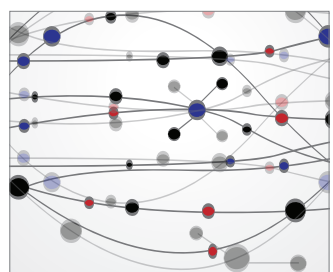

\section{The Scientific} World Journal
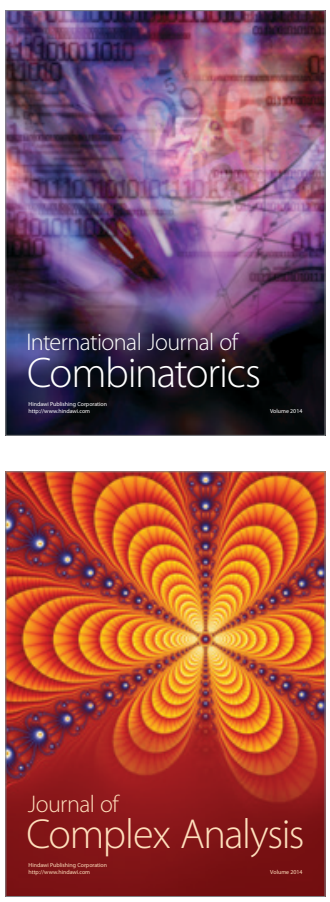

International Journal of

Mathematics and

Mathematical

Sciences
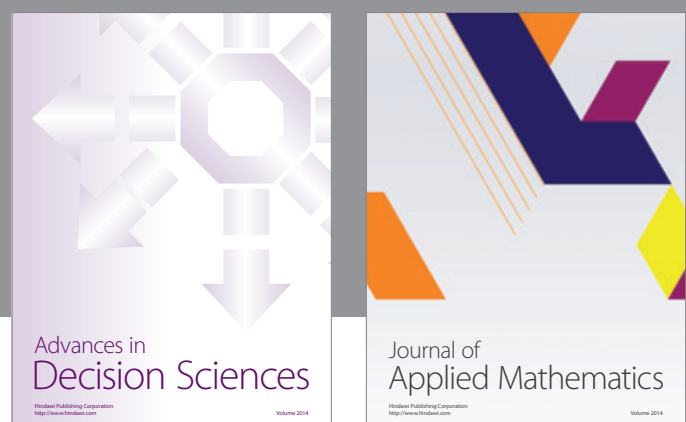

Journal of

Applied Mathematics
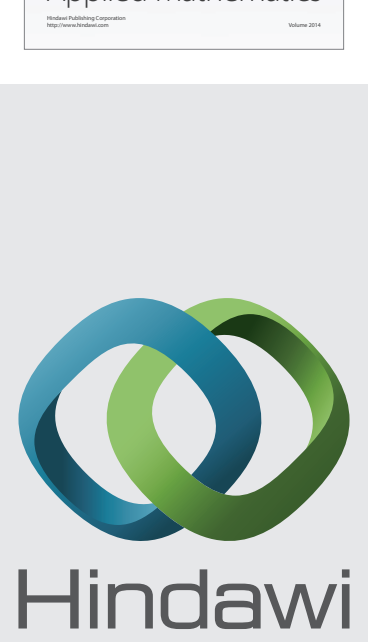

Submit your manuscripts at http://www.hindawi.com
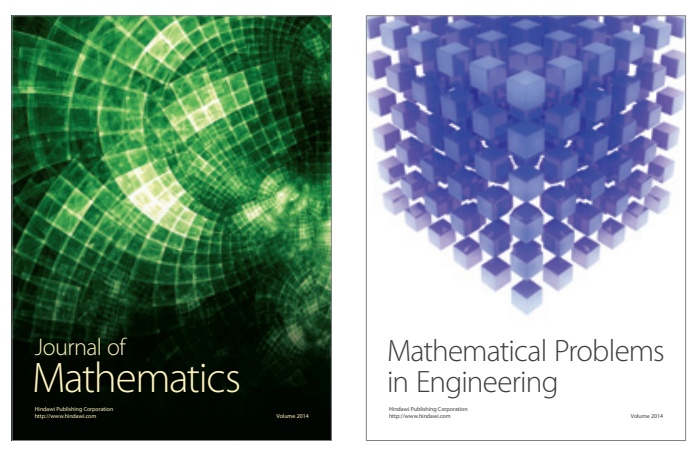

Mathematical Problems in Engineering
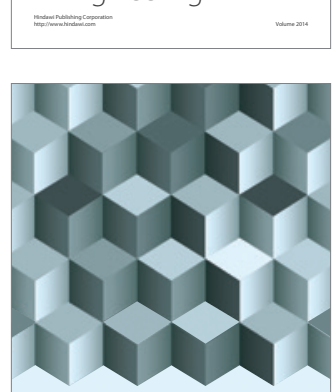

Journal of

Function Spaces
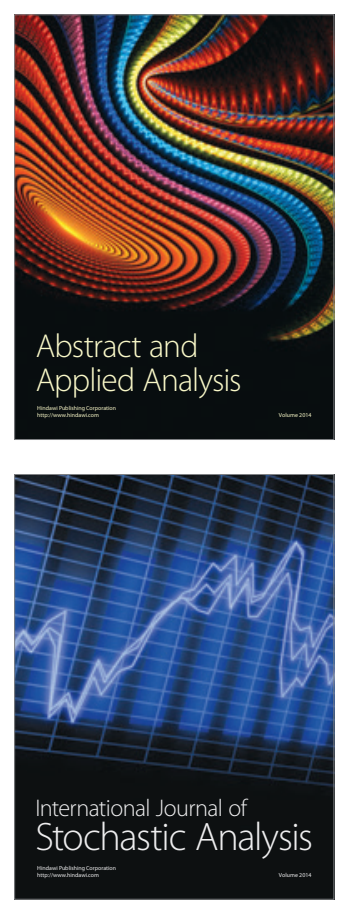

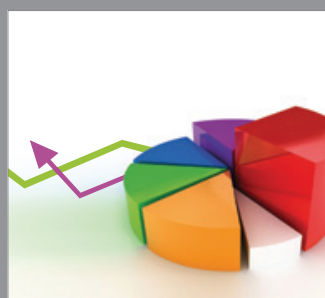

ournal of

Probability and Statistics

Promensencen
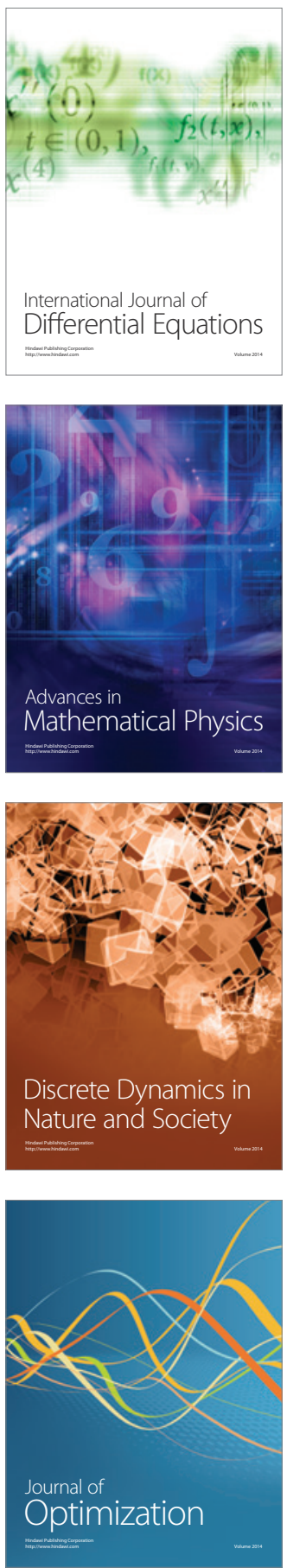